\title{
SUAV - SISTEMA UBÍQUO DE APRENDIZAGEM VERTICAL
}

\author{
Felipe Furtado Lorenci - UFSM - lorencifelipe@gmail.com; \\ Carmen Vieira Mathias - UFSM - carmenmathias@gmail.com; \\ Tiago Martinuzzi Buriol - UFSM - tiagoburiol@gmail.com.
}

\section{Resumo}

Muitos estudantes iniciam cursos do ensino superior com sérias dificuldades em tópicos de matemática, limitando seu desempenho em disciplinas como Cálculo e Álgebra Linear. Dessa forma, professores despendem um grande esforço para identificar estes problemas individuais e específicos, revisando os pré-requisitos necessários para cada conteúdo. Os dispositivos móveis são excelentes ferramentas quando usadas como plataformas de entrega de um ensino mais personalizado, porém, grandes potenciais dessas tecnologias ainda não foram completamente explorados. Este relato de pesquisa apresenta o desenvolvimento de uma aplicação para smartphones, destinada ao estudo de matemática apenas com a resolução de exercícios. A ideia central é que a partir de questões propostas, o aplicativo identifique as dificuldades do aluno, fornecendo dicas e materiais instrutivos relativos à essas deficiências; além disso, com uma análise nas respostas fornecidas pelo usuário juntamente com a frequência do uso de dicas, o sistema é capaz de reconhecer quais temas já estão dominados e também aqueles que ainda possuem lacunas, permitindo a criação de um mecanismo de identificação de pontos falhos e entrega de materiais específicos de forma clara, objetiva e orientada a cada usuário.

Palavras-chave: aprendizagem móvel, educação à distância, aprendizagem vertical, dispositivos móveis, tecnologia da informação.

\section{SUAV - UBIQUITOUS SYSTEM OF VERTICAL LEARNING}

\begin{abstract}
Many students face difficulties in topics of mathematics when they begin their undergraduate studies. Consequently, it can affect their performances in courses like Calculus and Linear Algebra. Thus, instructors have been struggling to identify these individual and specific difficulties in order to review prerequisites for each course topic. Mobile devices are excellent tools when used as delivery platforms to personalize teaching, but the whole potential of this technology has not been extensively studied. This research report presents the development of an application for smartphones designed to mathematics study with key-answers to exercises. The idea is that it can identify student's difficulties and provide tips and instructional materials to its users. Besides that, by analyzing the answers and the frequency of accessed tips, the system can recognize topics that are well known to students and topics that students face difficulties learning. Thus, it allows the development of a mechanism to identify points of failure and it provides specific materials in a clear, objective and oriented approach for each user.
\end{abstract}

Keywords: mobile learning, distance education, vertical learning, mobile devices, information technology. 


\section{Introdução}

Muitos estudantes iniciam cursos no ensino superior enfrentando dificuldades em tópicos que envolvem matemática, limitando seu desempenho em disciplinas como Cálculo e Álgebra Linear. Neste contexto, professores têm se esforçado para suprir e descobrir a origem deste problema, avaliando como os conteúdos estudados no Ensino Médio refletem em um bom desempenho no Ensino Superior; de forma mais precisa: por que alguns alunos sofrem impactos tão fortes ao ingressar em cursos das Ciências Exatas e Tecnológicas? Quais conteúdos de Matemática deveriam ser tratados de forma mais concisa no Ensino Médio, para que os alunos não chegassem com defasagens tão grandes no Ensino Superior? E ainda: como preencher essas lacunas de forma efetiva, levando em consideração o tempo disponibilizado para a disciplina no Ensino Médio?

Trabalhando com alunos calouros de uma universidade federal do sul do país, nota-se que é grande o índice de evasão nas disciplinas que dependem de conhecimentos matemáticos prévios, independentemente do curso analisado. Uma das disciplinas que os alunos apresentam considerável dificuldade é o Cálculo, que em particular, nessa instituição, é comprovada por Molon (2013) ao apresentar os índices de não aprovação no período de 2009 a 2012, que atingiram o índice de 58\%, para as turmas que cursam regularmente a disciplina de Cálculo I.

Tal disciplina, tem como pré-requisitos conhecimentos de matemática elementar, funções, trigonometria e conceitos de geometria plana e espacial. Esta última, tem sido um problema comum à vários alunos, pois em alguns casos, quando vista no Ensino Médio, é trabalhada de forma sucinta e inconsistente; assim, o aluno não realiza o processo de construção do conhecimento como deveria, deixando lacunas em tópicos que seriam essenciais para suas disciplinas subsequentes. Cury e Bisognin (2006), afirmam que grande parte das dificuldades encontradas nos conceitos que envolvem geometria, são de fato, trazidas do Ensino Básico. Corroborando esta ideia, Balomenos, FerriniMundy e Dick (1994), defendem que muitos dos conhecimentos necessários para uma compreensão do cálculo no Ensino Superior, são embasados no curso tradicional de geometria do nível básico.

Levando em consideração o atual paradigma tecnológico, muitos professores vêm reconhecendo as Tecnologias da Informação e Comunicação (TIC) como preciosas ferramentas de apoio ao ensino, sobretudo, quando vinculadas à dispositivos móveis, como smartphones e tablets, que estão cada vez mais presentes no cotidiano juvenil. Segundo Wains e Mahmood (2008), o m-learning ${ }^{1}$ é uma realidade emergente que engloba tecnologias sem fio e computação móvel, permitindo que a aprendizagem ocorra a qualquer tempo e lugar, maximizando a liberdade dos alunos.

Estas plataformas, permitem um acesso rápido à informação, além de possibilitar um ensino personalizado e individualizado, suportando inúmeros objetos de aprendizagem e incorporando tecnologias não usuais na área da educação, tais como a realidade aumentada (Macedo, Silva e Buriol; 2016).

Neste contexto, este relato de pesquisa tem por objetivo apresentar o desenvolvimento de um aplicativo para dispositivos móveis voltado ao estudo de matemática baseado apenas na resolução de exercícios. A ideia central é que a partir de questões propostas pelo algoritmo, o aluno identifique suas dificuldades e receba materiais e conteúdos educacionais específicos. Com base no desempenho do usuário, o que inclui análise de erros, percentual de acertos e uso de dicas, o sistema tem a capacidade de identificar os temas já dominados, bem como aqueles que ainda necessitam

\footnotetext{
${ }^{1}$ Mobile learning - Aprendizagem por meio de dispositivos móveis.
} 
de mais estudo, permitindo a criação de um mecanismo inteligente de identificação de erros e preenchimento de falhas.

\section{Material e métodos}

A fim de entender e investigar as dificuldades mais pertinentes entre os alunos, no ano de 2016 os autores deste trabalho colheram dados em um Colégio Técnico da mesma cidade já citada. Esta instituição conta com diversos cursos, tanto integrados ao Ensino Médio, quanto subsequentes. Neste caso, priorizou-se os cursos integrados: Eletrotécnica, Informática e Mecânica.

Aplicou-se um teste composto por 10 questões, distribuídas em 3 níveis: 2 questões consideradas fáceis, 7 questões médias e apenas uma difícil. Para realizar esta classificação, levou-se em conta os conteúdos envolvidos, a lógica e o número de procedimentos que deveriam ser realizados para resolver o problema e como o enunciado da questão era disposto.

Em reunião com a escola, levou-se em pauta que seria de particular pertinência o trabalho com situações problema propostas no Exame Nacional do Ensino Médio (ENEM); assim, as questões foram extraídas desta prova, sendo retiradas as alternativas, da mesma forma que os enunciados foram modificados estrategicamente para que os alunos expusessem suas metodologias de resolução.

Os testes foram corrigidos de forma sigilosa e individual: por meio de uma tábua de algoritmos referência de resolução, cada prova foi examinada de forma única e direta, buscando-se traçar o perfil do estudante e os conteúdos de maior e menor domínio, seguindo a análise de erros proposta por Cury e Bisognin (2006), o que é de particular interesse para este projeto.

Além disso, entrevistou-se profissionais da rede pública e privada de ensino, onde expuseram os tópicos que os alunos possuem maior dificuldade, os que possuem um maior número de pré-requisitos e aqueles que comumente são menos priorizados, ou por vezes, não são trabalhados por conta da indisponibilidade de recursos e/ou tempo acadêmico. Munidos destes dados, iniciou-se a programação do aplicativo e a seleção das questões e materiais instrutivos, que até o presente momento, são focados na geometria espacial.

Em um primeiro momento, realizou-se uma varredura nas principais opções de ambientes de desenvolvimento integrado (IDE) voltados ao mobile development ${ }^{2}$; optouse pelo Intel XDK. A escolha foi feita seguindo os seguintes critérios: permite um rápido desenvolvimento, visto que a implementação é baseada em tecnologias da Web (HTML5, CSS, Javascript); possui suporte para a simulação da aplicação antes de sua compilação final; disposição de uma considerável quantia de materiais de referência disponibilizados na rede (XDK, 2017); e principalmente, pela possibilidade do desenvolvimento de aplicações híbridas e compilação multiplataforma, o que contempla a execução na maioria dos dispositivos móveis do mercado.

Em um segundo momento, utilizando-se do framework Bootstrap - que reduz consideravelmente o tempo de implementação da parte visual/responsiva do aplicativo modelou-se a interface básica, que consiste em uma página simples de recepção e outras três de funcionalidades: uma de informações sobre o projeto, outra de configurações e a página principal, dividida em uma aba primária, onde as questões são exibidas e atualizadas conforme o algoritmo determina, e uma secundária, onde o usuário pode conferir o seu desempenho.

\footnotetext{
${ }^{2}$ Desenvolvimento voltado à dispositivos móveis.
} 
A interface foi desenhada com o visual mais limpo possível, seguindo os princípios da Teoria da Carga Cognitiva (Santos, Tarouco; 2007), evitando distrações e com foco direto nas questões; a cor predominante do $a p p^{3}$, o azul ciano, foi escolhida com base nos estudos de Eva Heller (2016), onde cita que esta cor nos remete à simpatia, tranquilidade, harmonia, fidelidade, concentração e virtudes intelectuais.

Em um terceiro momento, implementou-se o banco de dados online, com campos voltados ao armazenamento das questões, enunciados, dicas específicas e demais configurações. Nesta etapa, também foram criados os arquivos de controle do servidor, responsáveis pelo interfaceamento entre os dispositivos móveis e o banco de questões: ao receber as requisições dos dispositivos, o algoritmo busca no banco e retorna para o solicitante as informações correspondentes, conforme ilustra a figura 1.

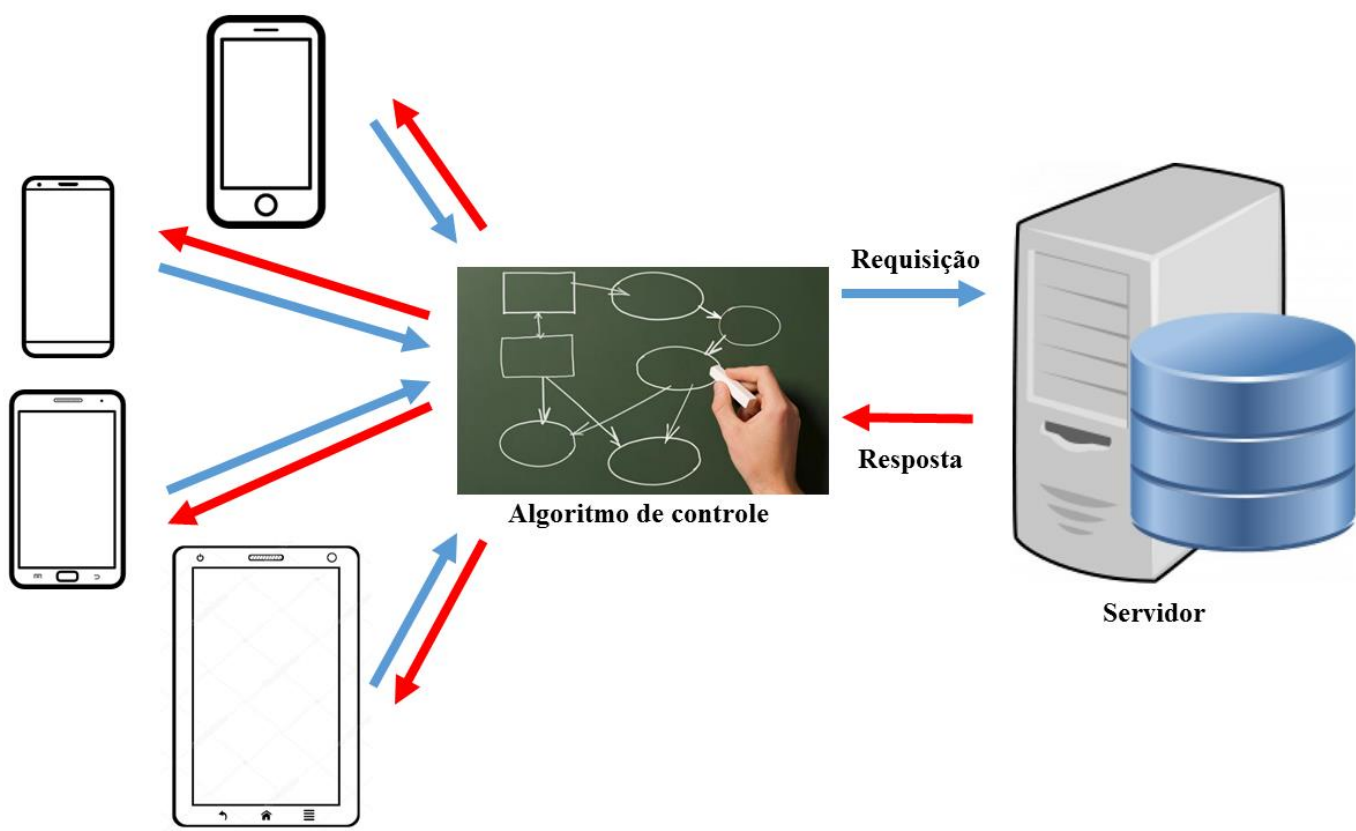

Figura 1 - Interface entre dispositivos e banco de dados

Testada esta funcionalidade, em um quarto momento focou-se na implementação da rotina cerne do aplicativo, que é responsável pela escolha da questão que o usuário deve realizar. Este algoritmo roda independentemente em cada dispositivo e analisa estatisticamente o desempenho de cada usuário.

As questões escolhidas, foram estrategicamente distribuídas no banco de dados em 3 níveis: fácil, médio e difícil, cada uma delas, contendo um único código de identificação (label). Ao inicializar o aplicativo pela primeira vez, o programa define automaticamente o nível atual como intermediário e a partir do progresso do aluno, requer ao banco de dados questões do nível mais adequado à situação atual; além disso, ao primeiro uso, o programa gera uma lista (array) de questões disponíveis para cada nível, de acordo com consulta ao banco de dados externo, salvando-as de forma persistente no dispositivo. Ao acertar uma questão, ela é retirada da lista; caso contrário, ela permanece.

Em um primeiro momento, por circunstâncias de teste de mesa, programou-se a rotina de tomada de decisões da seguinte forma: acertando-se três questões consecutivamente, sem o uso de dicas, o usuário passa para o próximo nível, onde continua seu progresso. Caso o usuário acerte as questões com o uso de dicas e materiais instrutivos, que são sugeridos pelo próprio aplicativo, ele continua realizando questões

\footnotetext{
${ }^{3}$ Aplicativo que pode ser executado em um browser.
} 
do nível em que se encontra, até que sejam acertadas todas as disponíveis - aí sim, ele passa ao próximo nível. Caso ele erre três questões consecutivas, independentemente do uso de dicas, ele regride de nível.

Por fim, realizou-se exaustivos testes de desempenho do aplicativo, a fim de aferir o tempo de resposta do servidor, a acuracidade do algoritmo de escolha de questões e se os dados estatísticos locais estavam sendo calculados e salvos de maneira correta.

Atualmente, está em fase de implementação uma versão atualizada do aplicativo, na qual está sendo inserida um sistema de pontuação, com o objetivo de dar um aspecto gameficado ${ }^{4}$ ao app, tornando-o mais atrativo aos alunos. Além disso, o banco de dados local está sendo aperfeiçoado, para que os usuários não fiquem dependentes de conexões com a internet para utilizar o serviço. Também, a interface está sendo otimizada, incluindo pequenas animações, o que ratifica a ideia da gamificação. Por fim, está sendo programado um sistema de retorno de dados estatísticos aos servidores, para que posteriormente, se tenha uma maior noção de como os alunos estão evoluindo com a aplicação.

\section{Resultados e discussões}

Tanto o questionário de triagem aplicado aos alunos da escola, quanto as entrevistas realizadas com professores do Ensino Médio convergiram para o mesmo ponto: uma dificuldade comum aos alunos é a Geometria Espacial. Ainda não há exatidão para a causa deste problema nas escolas estudadas, mas Bermejo (apud HOFFER, 1981), evidencia que estes déficits podem ser originários da ausência de trabalho com a geometria de posição e o desenho geométrico; do desuso, por parte de alguns professores, das representações de figuras planas e espaciais; da falta de trabalho com planificações de sólidos e da ausência, em alguns casos, de um trabalho voltado a percepção, que auxilia na representação mental dos objetos.

Assim, ao desenvolver um aplicativo para dispositivos móveis voltado ao ensino de geometria espacial, pretende-se sobretudo, disponibilizar mais uma ferramenta de aprendizagem aos alunos; com esta ferramenta, espera-se sanar as dúvidas específicas de cada usuário, uma vez que o aplicativo tem inteligência suficiente para reconhecer os pontos fracos de cada um, fornecendo tópicos instrucionais adequados, ajudando o aluno a superar estas dificuldades.

Em testes preliminares, a aplicação revelou-se como mais uma opção de material de apoio ao processo de ensino-aprendizagem, tanto para o aluno quanto para o professor. Por um lado, o aluno recebe materiais específicos por meio da prática continuada de exercícios, preenchendo lacunas que tradicionalmente ficariam em aberto. Por outro, o professor tem a segurança de que além do aluno estar exercitando os conteúdos trabalhados em sala, ele também estará fazendo isso de forma ativa e consciente, tendo noção da gênese de seus erros e também dos conteúdos que já estão dominados.

Do ponto de vista técnico, o aplicativo (figura 2) demonstra bastante eficiência na execução, também permitindo bastante facilidade para sua manutenção: projetado de forma modular, não se torna dispendioso alterar o algoritmo de controle principal, podendo-se a qualquer momento modificar os critérios de escolha das questões e seus níveis; por ter um banco de dados externo aos aparelhos, também permite constantes atualizações no banco de questões, sendo posteriormente sincronizadas de forma automática com os dispositivos.

\footnotetext{
${ }^{4}$ Gamificação - Uso de mecânicas e dinâmicas de jogos para engajar pessoas, resolver problemas e
} melhorar o aprendizado, motivando ações e comportamentos em ambientes fora do contexto de jogos. 


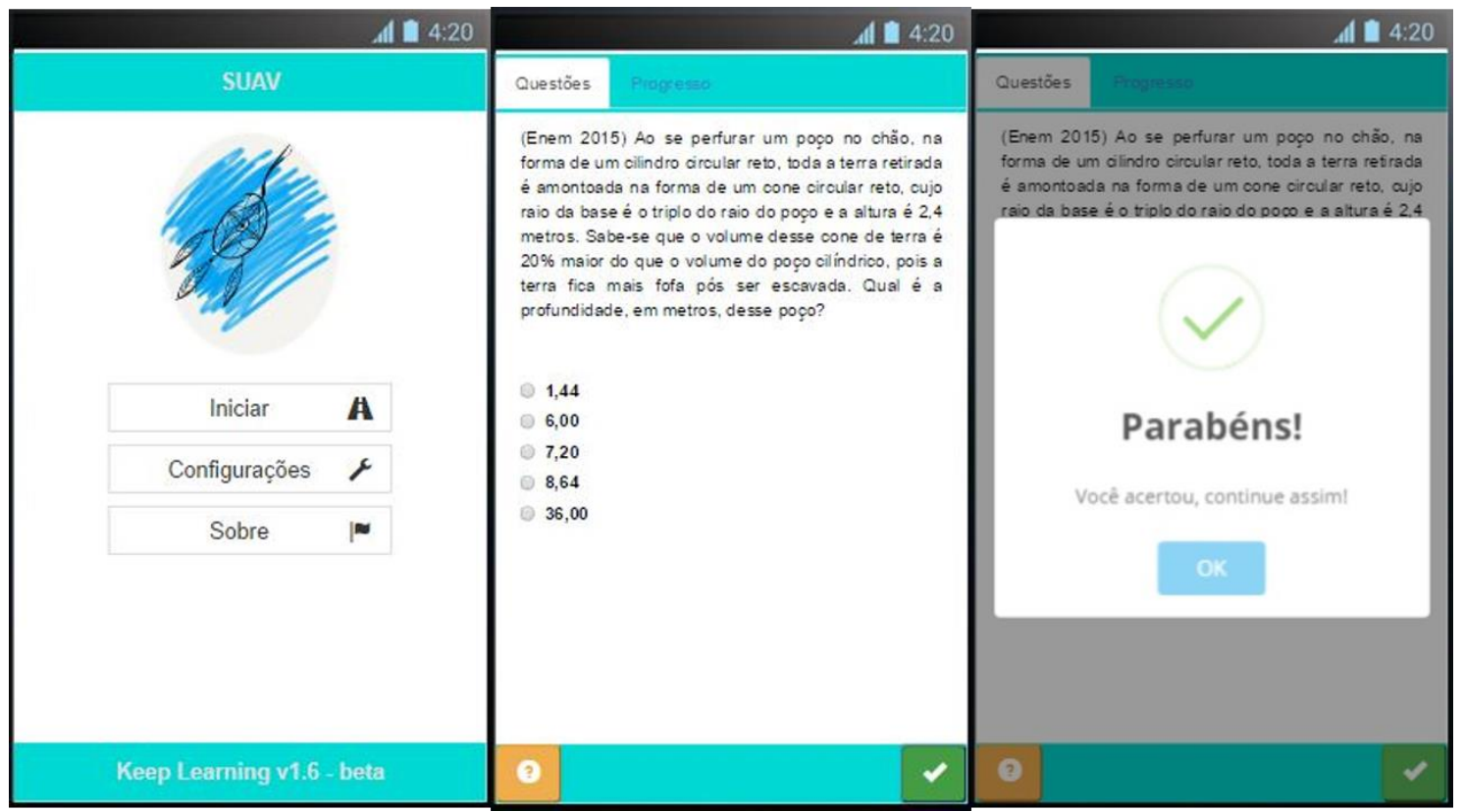

Figura 2 - Capturas de tela da aplicação em funcionamento

Com as alterações que estão sendo realizadas no aplicativo, a longo prazo, esperase que este objeto de aprendizagem também se torne uma rica fonte de coleta de dados estatísticos em larga escala, detectando informações valiosas sobre cada usuário, podendo futuramente beneficiar estudos em áreas afins, como análise da qualidade de ensino por região, desenvolvimento de novos algoritmos de inteligência artificial voltados ao ensino, análise qualitativa de erros e aprimoramento das TIC como um todo.

Alguns dados relevantes a ser armazenados de forma anônima, criptografada e com o aval do usuário, são a escola atual do aluno, a geolocalização, o tempo de resolução de cada questão, os acertos/erros mais frequentes, a periodicidade do uso de ajuda para resolver cada situação problema e quais tópicos de cada conteúdo são os maiores geradores de dúvidas e equívocos.

\section{Conclusão}

Dentro de nosso contexto tecnológico, torna-se inviável tratar a educação de forma alheia às TIC. Os smartphones e tablets permitem uma flexibilização da educação como ainda não se havia visto, possibilitando um rápido e universal acesso à informação em larga escala - qualquer um, tem acesso a qualquer dado, de qualquer local do globo, a qualquer hora.

Assim, neste trabalho relatou-se brevemente o desenvolvimento de um aplicativo para dispositivos móveis, com foco na área de geometria espacial. Espera-se que haja de fato, uma educação mais flexível e personalizada às necessidades de cada aluno, o que é um grande desafio aos professores. Também, espera-se que os usuários obtenham sucesso em provas seletivas ao ensino superior, uma vez que terão um acompanhamento exclusivo e individualizado.

Em um futuro próximo, pretende-se retornar à escola já citada, com o objetivo de testar a eficácia da atual versão do aplicativo: as turmas visitadas serão separadas em dois grupos: um controle, que receberá livros e materiais didáticos usuais para o estudo de geometria espacial, e outro, que usará apenas o aplicativo. Serão realizados testes com os dois grupos, anteriormente e posteriormente ao tempo de estudo, a fim de verificar qual das amostras obteve maior efetividade no processo de aprendizagem, considerando-se o mesmo período de tempo. 
Por fim, almeja-se popularizar ainda mais o uso de mídias e tecnologias da informação e comunicação voltadas ao ensino, visto que estas facilitam tanto o entendimento dos conteúdos, quanto suas formas de apresentação.

\section{Referências bibliográficas}

BALOMENOS, R., FERRINI-MUNDY, J. E DICK, T. Geometria: prontidão para o Cálculo. In M. Lindquist e A. Shulte (Org.), Aprendendo e Ensinando Geometria. (pp. 240-257). São Paulo: Atual Editora. 1994.

COSTA, C, A., BERMEJO, B, P, A., MORAES, F, S, M. Análise do ensino de geometria espacial. 10 f. X Encontro Gaúcho de Educação Matemática (EGEM), Ijuí - RS, 2009.

CURY, H. N., BISOGNIN, E., Calculando o volume de um sólido: como a análise de erros pode auxiliar professores a elaborar atividades de ensino para calouros de engenharia. Anais: XXXIV - Congresso Brasileiro de Ensino de Engenharia. Passo Fundo: UPF, 2006.

HELLER, E. A psicologia das cores - Como actuam as cores sobre os sentimentos e a razão. Barcelona - Espanha: Editorial Gustavo Gili, 2016.

HOFFER, A. Geometry is more than proof. Mathematics teacher. January, 1981.

INTEL XDK Reference. Intel, 2017. Disponível em: <https://software.intel.com/ptbr/intel-xdk/documentation>. Acesso em: outubro de 2017.

MACEDO, A. C., SILVA, J. A., BURIOL, T. M. Usando Smartphone e Realidade aumentada para estudar Geometria espacial. RENOTE - Revista Novas Tecnologias na Educação, Porto Alegre, dez. 2016. Disponível em: <http://seer.ufrgs.br/index.php/renote/article/view/70688>. Acesso em: dez. 2017.

MOLON, J. Cálculo no Ensino Médio: uma abordagem possível e necessária com auxílio do Software Geogebra. 2013. 195 f. Universidade Federal de Santa Maria, Santa Maria, 2013.

SANTOS, L, M, A., TAROUCO, L, M, R. A importância do estudo da teoria da carga cognitiva em uma educação tecnológica. RENOTE - Revista Novas Tecnologias na Educação, Porto Alegre, jul. 2007. Disponível em: <http://seer.ufrgs.br/renote/article/viewFile/14145/8082>. Acesso em: dez. 2017.

WAINS, S. I.; MAHMOOD, W. Integrating m-learning with e-learning. In: Conference on information technology education, 9., 2008, Cincinnati, OH, USA. Proceedings. New York, USA, ACM, 2008. p. 31-38. 